UC-4

Issued: November 1977

\title{
Determination of Nitrogen in Boron Carbide with the Leco UO-14 Nitrogen Determinator
}

R. D. Gardner

W. H. Ashley

A. L. Henicksman 
This work was sponsored by the Division of Reactor Development and Demonstration of the US Energy Research and Development Administration.

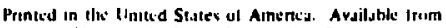
Nathosus lechnices Infurmation Service

ti.s. Ixiparimient of Cummecte

szks Port Huyal Roud

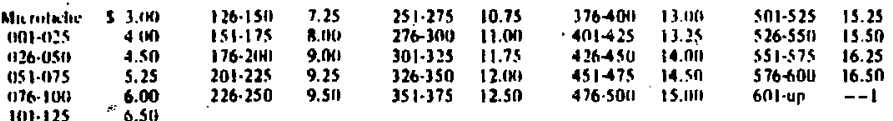

1. Add $\$ 2.511$ for each additional l00-page inctement from 601 pages up.

This report wes prepared as an wecciunt of wirk sponesred uy thi United Sistes Guvernment. Neither the United Stutrx nur the United States Department of knerny, nur any ut these -mployers, not any of thror contracturs, subcentreckars, or

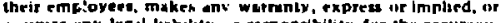

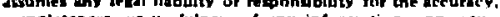

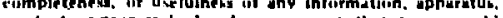

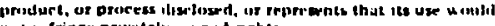
not in'ringe prisutely uwinod nghis. 


\title{
DETERMINATION OF NITROGEN IN BORON CARBIDE WITH THE LECO UO-14 NITROGEN DETERMINATOR
}

\author{
by
}

\author{
R. D. Gardner, W. H. Aghley, and A. L. Henicksman
}

\begin{abstract}
Use of various metals as fluxes for releasing nitrogen from boron carbide in the Leco Nitrogen Determinator was investigated. Metals such as iron, chromium, and molybdenum that wet the graphite crucible all promoted nitrogen release. Tin, copper, aluminum, and platinum did not wet the graphite and were of no value as fluxes. A procedure for sample handling and the resulting performance of the method are described. The precision at $0.06-0.6 \%$ nitrogen averaged $4 \%$ relative standard deviation.

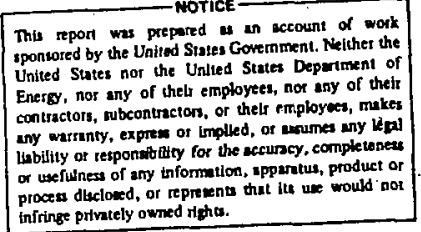

\section{INTRODUCTION}

Nitrogen in boron carbide has been determined by several different methods. In the classical Dumas method,' the sample is heated with copper oxide, the evolved nitrogen is purified by passage through potassium hydroxide solution, and the nitrogen is measured by volume. Other procedures are modifications of the $\mathrm{Kjeldahl} \mathrm{method} \mathrm{in} \mathrm{which} \mathrm{nitrogen} \mathrm{is} \mathrm{converted} \mathrm{to} \mathrm{ammonia}$ either by acid digestion at high temperatures ${ }^{2}$ or by fusion with lithium hydroxide. ${ }^{2,4}$ In either technique, the ammonia may be measured spectrophotometrically using Nessler's reagent" or titrated with standard acid.' All these methods have the same problem, the difficulty with which boron carbide is decomposed to release the nitrogen. Any of the methods works with boron nitride and with some batches of boron carbide. We have found great variation in the reactivity of different batches of boron carbide; some are completely decomposed, whereas others hardly are attacked. In using the Dumas method, we sometimes have found nitrogen still being evolved after $8 \mathrm{~h}$ of heating. In sealed tube digestion in sulfuric acid, dissolution sometimes is not complete after $16 \mathrm{~h}$ at $500^{\circ} \mathrm{C}$. At least, when using this procedure, one can see whether dissolution is complete before proceeding with the analysis. In using the alkaline fusion method, the recommended way to ensure that a given treatment is adequate for a given batch of boron carbide is to get concordant values from samples of widely varying sizes.

A Leco Nitrogen Determinator, U0-14, was tried at the Hanford Engineering Development Laboratory (Method 40.6, Ref. 3). This instrument, marketed by the Leco Corporation of St. Joseph, Michigan, uses an impulse furnace to heat the sample in a graphite crucible under a helium atmosphere. The nitrogen released from the sample is separated from other gases by a combination of chemical absorbants and a refrigerated column of molecular sieve. The nitrogen is detected by thermal conductivity. This signal is amplified, integrateci, and displayed on a 
digital voltmeter. We got one of these instruments, but were not immediately successful in using it to determine nitrogen in boron carbide. The sample treatment that finally gave acceptable results, and the reliability of the method, are described here.

\section{RECOMMENDED PROCEDURU}

\section{A. Appáratus}

Balance, Analytical, capable of weighing to $0.01 \mathrm{mg}$.

Nitrogen Determinator, Leco, UO-14, Model 875-631, with necessary electrical, water, and helium services.

Hreas, Laboratory, hydraulic, capable of $100-\mathrm{MPa}$ pressure, with punch and die for forming 6 mm-diam cylinders.

Shatter Bax, with tungsten carbide grinding assembly, from Spex Industries, Inc., 3880 Park Ave., Metuchin, NJ.

\section{B. Reagents}

Abaorbere and Deolccants as furnished with the instrument.

Helium, high-purity

Iron, powder, nitrogen-free.

Nickel, 0.025-mm sheet, nitrogen-free.

\section{Standards}

Callbration Standarde, NBS-SRM steels certified for nitrogen, NBS-SRM 160 or 10; or Leco Nos. 501-551, 501-552, and 501-553.

Control Standard. A powdered sample of $\mathrm{B}_{4} \mathrm{C}$ that has been analyzed repeatedly by other methods is helpful for establishing that the method is under control.

\section{Sample Preparation}

1. Pulverize the sample in the Shatterbox.

2. Weigh $500 \mathrm{mg}$ of iron powder onto the balance pan.

3. Weigh $10-50 \mathrm{mg}$ of sample onto the iron powder.

4. Carefully mix the two powders and transfer the mixture to a nickel capsule made by rolling a 25- by $35-\mathrm{mm}$ rectangle of nickel sheet around a $5-\mathrm{mm}$-diam glass stirring rod and pinching and folding one end shut. 
5. Close the other end of the capsule and press it into a compact peliet using a hydraulic laboratory press with a $6-\mathrm{mm}$-diam punch and die at about $70-\mathrm{MPa}$ pressure.

\section{E. Operation}

1. Prepare the instrument for analysis as directed in the Instruction Manual.' If the instrument is in "standby" condition, preparation involves only turning on the refrigeration unit to let the irap temperature drop to about $2^{\circ} \mathrm{C}$ and making an electronic check to ensure that the bridge output is zero and that the DVM reading remains steady when the "Function Select" switch is in the "Integ. Check" position. If the power has been turned off, let the instrument warm up for several hours with the helium flowing before making any checks. The bridge output will drift continually until all temperatures are at equilibrium.

2. Run several known samples and make any final adjustments in the electronics or timing. (The timing is best checked with a strip-chart recorder and best regulated by. means of the measured gas flow or the temperature of the molecular sieve trap.) Leave the Blank Adjust on 0, the Slope Adjust on 5, and the Weight Compensator on 0.500 .

3. Run at least three prepared portions of each sample with at least three standards and three blanks mixed into the set so that any instrument drift will be averaged out. If Leco standards are used, weigh them rather than just accepting them as being $0.500 \mathrm{~g}$. Two types of blank must be run, an tmpty crucible for the standards, and, for the samples, a pressed pellet made from a nickel capsule containing $500 \mathrm{mg}$ of iron powder.

\section{F. Caiculations}

$$
N_{2}=\frac{v_{2} F}{w_{2}}
$$

where

$$
\begin{aligned}
N_{2}= & \text { per cent of nitrogen in sample } \\
V_{2}= & \text { DVM reading for sample minus rearing } \\
& \text { for blank } \\
W_{2}= & \text { weight of sample in grams } \\
F= & \begin{array}{l}
\text { average of } F \text { values calculated below for } \\
\text { standards. }
\end{array} \\
F= & \frac{W_{1} N_{1}}{V_{1}}
\end{aligned}
$$

where

$$
\begin{aligned}
F= & \text { factor for calculations above } \\
W_{1}= & \text { weight of standard in grams } \\
N_{1}= & \text { per cent of nitrogen in standard } \\
V_{1}= & \text { DVM reading for standard minus } \\
& \text { reading for blank. }
\end{aligned}
$$




\section{EXPERIMENTAL}

\section{A. Use of Strip-Chart Recorder}

It is very important that the chromatographic peak due to the nitrogen in the sample occur entirely within the integration period of the digital voltmeter. Figure 1 shows the effects of poor timing. Any part of the integral peak that lies outside the integracion period is lost, and, because of a characteristic of the digital voltmeter, any part below the level at the start of the integration period is subtracted. The timing of the electronics is controlled by variable resistors on the circuit cards, and once set properly at the factory it should not require adjustment. The timing of the nitrogen peak, however, may change owing to variations in the gas flow or in the temperature of the trap or the chromat graphic column. It may need regulation.

\section{B. Use of Metal Fluxes}

Heat alone in the Leco Determinator did not release any nitrogen from boron carbide. Because a platinum bath is commonly used in determination of gases in metals, we tried that approach, but the boron carbide floated to the top of the platinum. Some metals, when melted in the graphite crucible, wet the crucible and reacted with it. Others simply formed a ball that did not adhere to the graphite. On the hypothesis that metals which wet graphite would also wet boron carbide, we checked several metals for this property. Those that did wet the crucible included iron, nickel, chromium, vanadium, and molybdenum. All also seemed equally effective in releasing nitrogen from boron carbide. Metals that did not wet the crucible or promote nitrogen release included tin, platinum, aluminum, and copper. Tin had a noticeably deleterious effect on nitrogen recovery even when used with one of the metals in the first series. This effect, and the advantage of intimate contact, are shown by the following data.

Using a sample of boron carbide, previously shown by our sealed tube method to contain $0.290 \%$ nitrogen, we made a series of runs in the Determinator by adding $0.5 \mathrm{~g}$ of nickel powder to the sample and placing it in a Leco tin capsule without mixing or pressing. The nitrogen found averaged $0.080 \pm 0.038 \%$. In a similar set, the sample was mixed with $0.5 \mathrm{~g}$ of iron powder, placed in a till capsule, and subjected to 5 metric tons of pressure. The average result of these runs was $0.138 \pm 0.029 \%$. In the next set, the sample was mixed with iron powder and placed in a nickel capsule, but not pressed. The average result was then $0.266 \pm 0.013 \%$. The final set was the same except that the hydraulic press was used again. The average result was $0.285 \pm 0.007 \%$.

INTEGRATION PERIOO

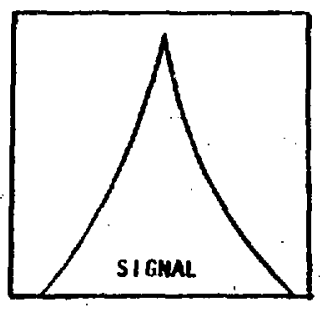

CORRECT TIIING

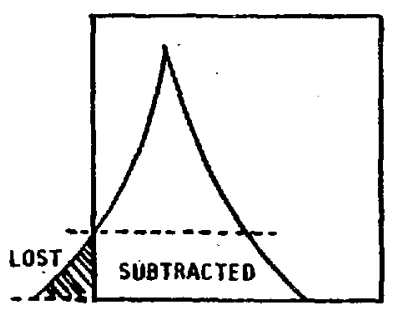

SIGKAL EARLY

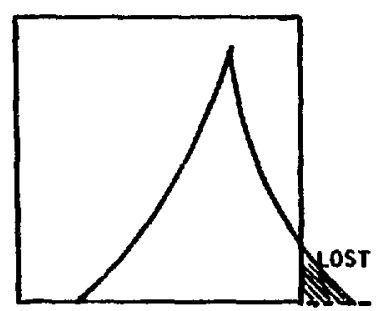

SIGMAL LATE

Fig. 1. ,

The effects of timing on the digital voltmeter output. 
Iron was chosen as the metal to mix with the powdered sample because it is readily available in powdered form with a low blank. Nickel foil was chosen for making capsules because it is ductile enough to be pressed without rupturing.

\section{RELIABILITY}

The conventional way to evaluate the performance of a procedure is to analyze certified samples, but in this case no such samples were available. We compared results obtained by this procedure with those of other reliable procedures, using a wide range of boron carbide materials. This comparison is shown in Table I. The precision shown is in absolute deviations. Over the whole range, the relative standard deviation is about $4 \%$. For each batch of material, there is an overlapping of individual results from the Leco and sealed tube ${ }^{2}$ methods, but the averages from the Leco procedure are consistently slightly lower. This lowness probably indicates less than $100 \%$ recovery of the nitrogen, but the loss is small enough to be acceptable in any but the most accurate work. The savings in time and material seem to favor the Leco Determinator for routine analyses.

\section{TABLE I}

\section{RESULTS OF DIFFERENT METHODS}

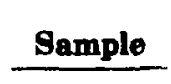

LA-1

B.C-32

B,C.51

BCPE-4A

BCPE-6-2A

BCPE-13A

Blend No. 3

\section{Dumas Method}

$(6) * 0.089 \pm 0.010 \%$

(8) $0.360 \pm 0.006 \%$

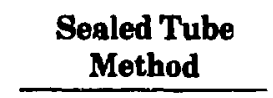
(5) $0.096 \pm 0.002 \%$
(4) $0.604 \pm 0.016 \%$
(7) $0.226 \pm 0.011 \%$
(12) $0.063 \pm 0.004 \%$
(10) $0.131 \pm 0.004 \%$
(7) $0.372 \pm 0.005 \%$
(8) $0.362 \pm 0.015 \%$

\section{Leco Method}

(10) $0.092 \pm 0.005 \%$

(12) $0.576 \pm 0.020 \%$

(12) $0.213 \pm 0.008 \%$

(10) $0.060 \pm 0.004 \%$

(10) $0.117 \pm 0.004 \%$

(8) $0.362 \pm 0.018 \%$

(8) $0.348 \pm 0.009 \%$

-The number of determinations in each set is shown in parentheses.

"Numbers following \pm signs are aboolute standard deviations. 


\section{REFMRENCES}

1. W. F. Hillebrand, G. E. F. Lundell, J. Hoffman, and M. S. Bright, Applied Inorganic Analysis, 2nd Ed. (John Wiley \& Sons, New York, 1953), pp. 779-83.

2. R. E. Perrin, A. Zerwekh, and W. H. Ashley, "Sealed-Tube Dissolution Method for Determination of Nitride Nitrogen in Boron Carbide," Los Alamos Scientific Laboratory report LuA5672 (July 1974).

3. Analytical Chemistry Laboratory Manual, Vol. 3, "Analytical Chemistry Methods for Boron Carbide Absorber," Method 40.7, Hanford Engineering Development Laboratory internal document (December 1975).

4. "Determination of Nitride Nitrogen in Boron Carbide by a Caustic Fusion Method," Los Alamos Scientific Laboratory internal document (March 1976).

5. Instruction Manual, UO-14 Nitrogen Determinator, Model 875-631, Leco Corporation, St. Joseph, Mich. 1976. 